Edith Cowan University

Research Online

ECU Publications Post 2013

$8-31-2020$

\title{
Mentalisation Amongst Maternal and Child Health Nurses Using the Newborn Behavioural Observations With Infant-mother dyads: A Qualitative Study
}

\author{
Kim Simkin-Tran \\ Edith Cowan University \\ Bronwyn Harman \\ Edith Cowan University \\ Susan Nicolson
}

Follow this and additional works at: https://ro.ecu.edu.au/ecuworkspost2013

Part of the Nursing Commons, and the Psychology Commons

10.1016/j.pedn.2020.01.018

(C) 2020. This manuscript version is made available under the CC-BY-NC-ND 4.0 license

http://creativecommons.org/licenses/by-nc-nd/4.0/

Simkin-Tran, K., Harman, B., \& Nicolson, S. (2020). Mentalisation amongst maternal and child health nurses using the newborn behavioural observations with infant-mother dyads: A qualitative study. Journal of Pediatric Nursing, 53, 21-27. https://doi.org/10.1016/j.pedn.2020.01.018

This Journal Article is posted at Research Online.

https://ro.ecu.edu.au/ecuworkspost2013/8777 
(C) 2020. This manuscript version is made available under the CC-BY-NC-ND 4.0 license http://creativecommons.org/licenses/by-nc-nd/4.0/ 
Mentalisation amongst Maternal and Child Health Nurses using the Newborn Behavioural Observations with Infant-Mother Dyads: A Qualitative Study

\section{Background}

Maternal and Child Health Nurses (MCHNs) are highly qualified in perinatal, infant and maternal mental health. They are often infant-mother dyads' first encounter with professional support following hospital discharge. Support extends through to school age and includes health surveillance, infant development guidance, and infant-mother early relationship promotion (Kemp, Anderson, Travaglia, \& Harris, 2002). This presents MCHNs a unique opportunity to promote dyadic bonds through long-term and collaborative practitioner-client relationships (Beam, O’Brien, \& Neal, 2010; Simpson, Condon, Price, Finch, Sadler, \& Ordway, 2016; Tomlin, Sturm, \& Koch, 2009). At times MCHNs must sit with emotional ambiguity and discomfort whilst holding space for dyads to navigate early relationships, a skill requiring mentalisation (Kemp et al., 2002; Tomlin et al., 2009).

\section{Mentalisation}

Mentalisation is an important, often preconscious, intra-psychic skill whereby individuals interpret and give meaning to their own thoughts, feelings, intentions, and behaviours, as well as others (Bateman \& Fonagy, 2012; Fonagy, Steele, Steele, Moran, \& Higgitt, 1991). It requires skilled navigation through the complex interplay of conflicting views, values, and beliefs (Cologon, Schweitzer, King, \& Nolte, 2017). Mentalisation is precursory to verbal and behavioural decisions towards others (Shai, Dollberg, \& Szepsenwol, 2017; Simpson et al., 2016). Disrupted dyadic bonds are linked to physical and mental health problems later in infants' lives; maternal mentalisation is crucial to mediating this risk (Fonagy, Steele, Steele, Moran, \& Higgitt, 1991; Garner, 2013). 
Mentalising mothers can interpret infant cues accurately and are conscious of the separateness of their own thoughts with their infants' whilst also recognising the interaction between one another's mental states (Shai et al., 2017). Compromised mentalisation capacity may lead to disrupted emotional regulation, dissatisfaction in the parenting role, and disconnected infant-mother relationships (Burkhart, Borelli, Rasmussen, Brody, \& Sbarra, 2017; Rostad \& Whitaker, 2016; Suchman, Ordway, Heras, \& McMahon, 2016). Nurse delivered mentalisation-based interventions have been linked to improved maternal mentalisation (Ordway, Sadler, Dixon, \& Slade, 2014; Suchman et al., 2016).

MCHNs who employ mentalisation-based practice are able to reflect on their own thoughts and emotions and differentiate them from those of the dyad; the experience and needs of the infant and mother become the focus and the support offered extends from there. This mentalising process, of holding the dyad in mind, enables MCHNs to bring infants' mental states to life for mothers and help mothers overcome anxieties and develop mentalisation about their infant for themselves (Slade, 2006). MCHNs can model appropriate infant-mother interaction and validate existing dyadic relational strengths. With such support, mothers increase attentiveness towards their infants' mental states, linking them to behaviours and needs (Slade, 2006).

Mentalising MCHNs also experience reduced burnout risk due to increased emotional satisfaction, self-awareness, and empathy towards families (Beam et al., 2010; Kalliath \& Morris, 2002; Watson, Bailey, \& Storm, 2016). However, the time between reflective supervision sessions, workload, and schedules, present obstacles for the effective implementation of mentalisation-based practice (Ordway, McMahon, Kuhn, \& Suchman, 2018; Tomlin, Hines, \& 
Sturm, 2016; Watson et al., 2016). Interventions that support mentalisation must, therefore, be brief and easily incorporated into routine practice.

\section{The Newborn Behavioural Observations system}

The Newborn Behavioural Observations (NBO; Nugent, Keefer, Minear, Johnson, \& Blanchard, 2007) system is a brief relational tool suitable for use with infants up to three months of age. With parental input, NBO practitioners read and interpret infants' behaviour through 18 neuro-behavioural observations that focus on capacity to habituate, motor behaviour, social behaviour, and consolability. The NBO can be completed within 15 minutes and can be integrated seamlessly into MCHN home visits and clinical consultations.

The NBO supports infant development and caregiver-infant relationships through its capacity to 'bring infants into the present', showcasing their individuality, competencies and complex social ability (Barlow, Herath, Torrance, Bennett \& Wei, 2018; Nugent, Bartlett, \& Valim, 2014; Nicolson, 2015). A Cochrane Review by Barlow and colleagues (2018) showed early evidence of the NBO's effectiveness in supporting infant development and parent-infant relationships within varying contexts. Practitioners report increased confidence to deliver individualised support, accurately addressing infants' immediate relational needs (Holland \& Watkins, 2015; McManus \& Nugent, 2011). Preliminary NBO research findings in the Australian context are positive (Nicolson, Carron, Newman, \& Campbell, n.d.), but research concerning its value and applicability specifically to Australian child and family health nursing is limited. With the exception of Schilling, Nicolson, and Ridgway (2018), there has been minimal exploration of the Australian practitioners' perspective, specifically their mentalisation processes. 


\begin{abstract}
Aim
This study aimed to understand MCHNs' experiences towards infants-mother dyads when using the $\mathrm{NBO}$, and their views regarding the $\mathrm{NBO}$ as a support for their own mentalisation processes. It explores whether MCHNs believe collaborative relationships are promoted, allowing them to promote positive dyadic relationships in the first three months.
\end{abstract}

\title{
Methods
}

\section{Design}

This qualitative study utilised an interpretative phenomenological approach which emphasises the significant interactions between MCHNs' sense-making and their experiential recollections (Smith, Flowers, \& Larkin, 2009; Smith \& Osborn, 2008). Double hermeneutics were key to interpretation and analysis of MCHNs' experiences; an interpretative phenomenological feature which recognises the interplay of MCHNs' meaning-making with that of the researchers' subjective interpretations of the MCHNs' experiences (Smith \& Osborne, 2008). Interpretative phenomenology's idiographic commitment also allows in-depth experiences to be linked to theoretical frameworks and literature, allowing for shared and divergent themes to be identified across individuals (Smith et al., 2009). This approach allowed for interpretation of the MCHNs' cognitive and affective experiences whilst using the NBO with dyads beyond superficial recollections.

\section{Participants}

Participants were purposively recruited from the Australian NBO register of trained practitioners. An 'invitation to participate' was emailed to $77 \mathrm{NBO}$ practicing MCHNs in the state of Victoria. Five responded to the first round of recruitment, four responded to the second round, and one was recruited through snowballing (Smith et al., 2009). Ten MCHNS (females; 
aged 31-66 years) were recruited in total; eight from metropolitan locations and two from regional localities. Eligibility was based on past completion of NBO training and clinical use of the system within the previous twelve months.

\section{Data Collection}

Information letters and consent forms were emailed to participants and face-to-face or Voice over Internet Protocol (VoIP) interviews were scheduled. These forms were explained prior to interviews and participants were given opportunities to ask questions. Participants completing face-to-face interviews $(n=4)$ signed their consent forms, and participants completing VoIP interviews $(n=6)$ provided audio-recorded consent confirming agreement with the research conditions. Interviews were semi-structured and participant led, using an interview schedule comprising open-ended questions and exploratory cues to explore points of interest (refer to Table 1). Participants were first asked questions relating to eligibility and demographics. Questions following this related to experiences before and after receiving the NBO training, whilst using the system with dyads, and after integrating the NBO into their practice. To conclude, participants were advised that interviews would be transcribed and a summary of findings would be available following analysis. Contact details for the research team and an independent person were provided should participants have queries or concerns following interviews. Interviews ranged from 30 to 60 minutes. The study was conducted from February 2018 to October 2018. 
Table 1

Interview Schedule

\section{Questions}

Demographic information.

Please tell me about your practice as a MCHN prior to receiving NBO training.

Please describe your thoughts and experiences of receiving NBO training.

Please tell me about a recent experience where you used the NBO with an infant (aged 0-3 months), with their mother present.

Please describe your experience administering the NBO to the infant.

Please describe your observations of the mother throughout the appointment.

How do you think the NBO will fit into your future practice and your work with infantmother dyads?

\section{Exploratory cues}

*Age.

*Highest qualification.

*Years of practice.

*Positives/negatives of role/practice.

*Areas for improvement.

*Feelings towards clients and vice versa.

*Benefits/downsides of NBO training.

*Has your practice changed? How?

*What was the setting and who else was present?

*How did you feel while you were administering the NBO?

*What went well/not well?

*Feelings towards the infant and vice versa.

*What went well/not well?

*Feelings towards the mother and vice versa.

*Thoughts regarding mothers' feeling/thinking whilst the NBO was administered.

*Will you use the NBO in future practice?

*What benefits do you foresee for your practice and dyads?

Table 1 Interview Schedule

\section{Data Analysis}

Following interview transcription, interpretative phenomenological analysis was employed to reflectively engage with the MCHNs' experiences (Smith et al., 2009). The chief investigator analysed each interview individually; recordings were listened to in conjunction with first readings of transcripts. Transcripts were then re-read, allowing for data immersion and 
familiarisation. Excerpts of interest were highlighted with accompanying exploratory comments in a column labelled as such. Comments related to the participants' description of significant recollections, their use of language, and linkages to broader concepts. From this, emergent themes were identified and noted in a column labelled as such. Linkages between themes were drawn, forming themes and sub-themes. This process was repeated for each transcript.

Themes and excerpts from all transcripts were extracted and tabled; points of convergence and divergence were identified informing the reconfiguration of themes and subthemes. Transcripts were continuously reexamined throughout this process to determine the inter-relatedness of MCHNs' experiential accounts. Triangulation was also employed to safeguard participant autonomy and enhance interpretative rigour and validity (Yardley, 2008). Unannotated copies of all transcripts were provided to another researcher who independently conducted her own analysis. Their analysis was compared to that of the chief investigator's, differences in themes and sub-themes were discussed and adapted until consensus was achieved. Overall the cross-analysis yielded minimal discrepancies and variances were easily resolved. A summary of findings was emailed to all participants with a request for review and feedback, no MCHNs expressed disagreement with the findings.

\section{Ethics and Rigour}

This research was approved by the Edith Cowan University Human Research Ethics Committee. Participation was voluntary and presented no risks to the MCHNs. For confidentiality purposes, participants were assigned unique identifiers prior to their interviews. MCHNs were advised that their interviews would be audio recorded and electronically filed against their unique identifier in a password locked folder; any hard copies resulting from this study would be stored in a lockable filing cabinet. MCHNs' names were replaced with 
pseudonyms and identifying information was removed from transcripts. Participants were able to refrain from answering any questions, and study withdrawal was acceptable until 1 October 2018.

The chief investigator maintained a journal throughout the study; this enabled her to bracket her own cognitions and biases; thus, limiting her impact on the interviews and data analysis. The journal also provided a resource for themes to be checked against whilst allowing them to remain with the data and the MCHNs' experiences (Pietkiewicz \& Smith, 2014).

\section{Results}

The data analysis produced four themes: reflections regarding the dyad, personal reflections, reflection into action, and professional identity and future practice. Twelve subthemes illustrating MCHNs' progression through the mentalising process whilst delivering the NBO to dyads were identified.

\section{Reflections regarding the Dyad}

The MCHNs' initial reflections regarding infants' and mothers' feelings, thoughts and behaviours reflected the foundations of mentalisation as described by Fonagy and colleagues (1991). Sub-themes included initial observations and meaning-making of infants' unique capabilities ('seeing and feeling as the infant'), mothers' communication and behaviours ('seeing and feeling as the mother'), and the interactions between infants and their mothers ('seeing the infant-mother dyad').

Seeing and feeling as the infant. When reflecting upon pre-NBO practice, some MCHNs referred to infants abstractedly (e.g. 'the baby' and 'it'); others described infants as secondary to consultations with the primary participants being mothers. When describing their NBO practice, MCHNs' focus shifted towards infants as competent and sociable beings (Nugent, 
2015). Infants were referred to by gender or name, and MCHNs demonstrated sensitivity towards infants' complexity and individuality. They described feeling increased self-confidence and were able to move beyond superficial observations towards understanding the infants' behavioural motivations (McManus \& Nugent, 2011; Slade, 2006). Irene demonstrates this through her postNBO reflections of an infant who seemingly had feeding issues:

A disengaged baby may not feed well. It doesn't mean that she's a poor feeder, she can be quite depressed. That's something I've learnt in young babies. They will show us the way.

Seeing and feeling as the mother. Through NBO practice, MCHNs' were perceivably more attentive and empathic towards mothers, factors linked to decreased post-natal depressive symptomology (Horowitz, Murphy, Gregory, Wojcik, Pulcini, \& Solon, 2013). Abigail articulates her perception of one mother's struggle with adapting to parenting, highlighting the dissonance between the pre-parenting and parenting identity:

[Mum] wouldn't have a conversation with me regarding how she was feeling. When you went into [baby's] nursery, in [their] cot were all these photo frames of all these wonderful holidays that [their] mum and dad had had. I thought: "You're mourning your past life." The NBO was my port of entry with [mum].

Such in-depth reflections are precursory to appropriate corrective interventions when supporting mothers with complex needs; assisting MCHNs to better interpret mothers' expectations of both the consultations and the practitioner-client relationship (Myors, Cleary, Johnson, \& Schmied, 2018).

Seeing the infant-mother dyad. The NBO's infant inclusive quality enables MCHNs' to be sensitive towards the infant and mother together. As consultations unfold, MCHNs begin to notice mothers' misinterpretation of infant cues, finding inconsistencies between mothers' verbalisations and the infant's observed behaviour. Post-NBO sessions the nurses observed positive dyadic relational shifts and the beginnings of maternal mentalisation. Improved caregiver mentalisation is an essential feature of increased parenting satisfaction, improved 
sensitivity to infant cues, and reduced anxiety (Burkhart et al., 2017; Rostad \& Whitaker, 2016;

Suchman et al., 2016). The NBO's potential is illustrated in Nicola's account of a mother's connection with her infant for the first time:

[Mum] was very fascinated by the social connection aspects, and particularly watching [infant] when [infant] was tracking the red ball and responding to the sound. I could tell by [mum's] movement; she was moving closer to [infant] and leaning over to [infant]. I thought that was quite a powerful session for [the dyad]. I stopped hearing the interesting terms like the 'fake cry' comment over time. [Mum] seemed to be able to actually look at her baby and use her baby's cues.

\section{Personal Reflections}

MCHNs' understanding of dyadic affect and behaviour informed the stance they adopted in practice ('the thoughtful MCHN'), they fostered hopes for the dyads ('the empathic MCHN'), and prepared to take action ('the practical MCHN').

The thoughtful MCHN. When reflecting upon their pre-NBO practice, many MCHNs communicated a sense of helplessness, role monotony, and low confidence despite their extensive expertise; this is consistent with McManus and Nugent's (2011) findings. They indicated that time restrictions and limited resources were barriers to effective dyadic support. As Hayley highlights, MCHNs struggled to empower mothers due to the lack of infant focus and individualisation for mothers:

I think [MCHNs] can be very efficient in our handling of babies when they're not our own babies; we forget that [they're] not. It should be perhaps a softer approach that we should use and remember that they are just very small babies.

By way of addressing the aforementioned gaps, MCHNs articulated a need for personalised, brief, flexible, and relationship-based interventions that provide dyads with opportunities to purposively and meaningfully interact.

The empathic MCHN. Practitioners who adopt a mentalisation approach are patientcentred (Davidsen, 2009). In this study, MCHNs were unanimously empathic in their dyadic 
support. Despite the time-limited and prescriptive nature of consultations, Charlotte expresses a sense of responsibility and protectiveness towards her dyads' relationships. She voices a desire to assist for the long-term:

[Some mums] have anxiety, PND, and unsettled babies. Then they have to travel quite far to get assistance. I wanted to see if there was something that I could do to help these mums. The NBO helps to bring out the individuality of the baby to [MCHNs] so that we can then help out the [dyad]."

The practical MCHN. Through mentalisation, MCHNs' appreciated the complex interactions between their dyads' thoughts and their own self-reflections; this informed planning towards personalised support (Slade, 2006). Barbara links a mothers' struggles to her own experience, and uses this to provide individualised support:

A lot of [mums] say "I don't know what normal looks like. I want my child to have friends, but I don't know how to talk to school mums." I say to them: "Don't worry there's four kids in our household, I'm not good with school mums either." But I can understand how they would feel, it's very isolating.

Barbara also reflects that, through NBO practice, sometimes the most appropriate action is no action at all: "The less you do, the more you see." The NBO provides practitioners with confidence to stop, observe and see infants' capabilities. Sharing this experience with dyads may proactively prevent concerns (i.e. maternal depressive symptomology; Nugent et al., 2014).

\section{Reflection into Action}

The mentalisation process concludes with MCHNs acting upon their meaning-making of both dyadic thought processes and their own reflections (Beam et al., 2010; Fonagy et al., 1991). MCHNs advocate for this infants' individuality ('giving voice to the pre-verbal infant'), they promote positive infant-mother interaction ('bringing the dyad into the here and now'), and their practice evolves towards collaboration ('shifting focus from expert to collaborator').

Giving voice to the pre-verbal infant. Understanding infants' perspectives provide MCHNs with an added dynamic to consultations; they reported increased consideration and 
accuracy towards infants. The NBO allowed nurses to advocate for the pre-verbal infant (Nugent, 2013), as evidenced by Fiona:

[The NBO is] being the voice for the baby when the mother is not that connected at that point in time. The uniqueness of the baby, the baby being an individual, and the baby being able to show its likes and dislikes and communicate those things. The infant hasn't got anyone else to speak for them.

Similarly, Irene describes her NBO practice as "seeing through the baby's lens", and Grace states that she seeks "permission" from the infant. MCHNs' ability to view infants as capable and competent beings allowed them to be responsive to infants' subtle cues and readiness, this is evidence of strong mentalisation ability (Slade, 2006).

Bringing the dyad into the here and now. The NBO offers dyads an opportunity to simply 'be' in the present, providing a safe space for infants to show their mothers their multifaceted competencies and social skills (Cologon et al., 2017; Nugent et al., 2014; Ordway et al., 2014). This is captured in Abigail's reflection regarding her continued NBO practice:

Once you show a mum, or dad, the capabilities of an infant and you explain to them and they see the infant's intelligence they are less likely to ignore the infant. They're more likely to have better reflective functioning.

By extension, Evelyn describes the mother in her dyad as: "being more accurate when she read the baby's behavioural cues" following NBO sessions. Holding the infant in mind allows parents to see and appreciate their child as aware and purposeful, thus fostering interactive and nurturing dyadic relationships (Burkhart et al., 2017).

Shifting focus from expert to collaborator. Practitioner mentalisation enables a shift from authoritative practice to collaborative partnerships; partnerships that may mitigate maladaptive transgenerational attachment (Myors et al., 2018; Ordway et al., 2014). As with Sanders and Buckner (2006), MCHNs believed the NBO to be effective towards building 
practitioner-client relationships and dyadic relationships through the promotion of practitioner sensitivity and curiosity. Evelyn summarises the NBO's relational benefits:

I think it draws you closer to the family emotionally. I think that they feel that you have a general interest in them because you go through those things together, and you all observe the baby together. It's an experience that we shared so far that nobody else has observed.

The NBO provides a "structure and a hierarchical thought process" (Abigail) which "does a lot for standardising how we approach relationship building between new mums and new babies" (Grace). Rather than delivering interventions ‘to’ dyads, the NBO enables MCHNs to observe infants, embody their pre-verbal cues and model suitable responses; working 'with' mothers and providing them with opportunities to develop their own parental embodied mentalisation (Simpson et al., 2016; Shai et al., 2017; Tomlin et al., 2009).

\section{Professional Identity and Future Practice}

MCHNs expressed feelings of accomplishment ('emotional satisfaction: journeying alongside infants and their mothers') and fulfilment in their work ('professional pride') from using the NBO. They described both barriers and strategies to overcome them ('incorporating the NBO: overcoming challenges').

\section{Emotional satisfaction: Journeying alongside infants and their mothers. MCHNs}

gained emotional satisfaction from the practitioner-client relationships, a factor which may decrease practitioner burnout (Watson et al., 2016).

As those babies grow up the relationship with them is incredible. They really know me, even if they haven't seen me for weeks. I'm like: "Do they really recognise me?" That's on a selfish note, but it's special for the babies that they know there's another adult in the world who cares for them. (Heather)

Their choice to utilise the NBO was validated by mothers' expressed positive impacts on the infant-mother relationship. 
I get all emotional about it [teary]. [Mum] said to me: "I love it when I come here because I feel like you've given us a boost." That to me was what the NBO gave, it gave [the dyad] a language. (Barbara)

Using the NBO, emotional satisfaction is encouraged through a 'give and receive' process for all parties. The mother gains an accurate understanding of her infant and is empowered by her child and MCHN. The infant is able to show his or her capabilities to both adults and receives interactions consistent with his or her cues.

MCHNs are also protected against emotional overinvestment. The NBO's perceived improvements to practitioner mentalisation allows MCHNs to deliver relational support alongside holding clients in mind, sitting with ambiguity, and recognising the self as autonomous to the dyad (Cologon et al., 2017; Davidsen, 2009; Kemp et al., 2002).

Professional pride. MCHNs communicated a sense of comradery and professional pride due to their unique positions; "there is no stigma attached to coming to MCHNs because everyone [engages], every mum and every child does" (Barbara). This provides MCHNs with opportunities to develop long-term practitioner-client relationships enabling them to continuously observe and support dyadic growth. For instance, "[dyads] trusted us and I thought that's very special and very humbling in a way. A privileged position" (Heather).

Many MCHNs felt that the NBO should be formally incorporated into practice, describing short-term to long-term benefits for fellow practitioners and clients. The NBO enables MCHNs to showcase their expertise beyond physical health checks:

I think it's a fantastic framework to use and I think if [the NBO] was a cross-service tool for all nurses I think it would really help the families in their relationship building. I think it would really improve the standing of the service in the community. I think there's still that perception that we just weigh and measure babies, we can really alter that perception from the very beginning by having a focus on emotional regulation and attachment, and all the really important things. (Grace) 
Incorporating the NBO: Overcoming challenges. Challenges to the NBOs'

incorporation into clinical practice were identified and possible resolutions were suggested.

Regional MCHNs or those who did not have contact with other NBO trained colleagues voiced a need for clinical or peer supervision:

I'm the only one up here that has done the training. There is no one to debrief with. I don't know if they have that in other places where they have clinical supervision, they can talk about things that happened when they implemented the NBO. I don't have that. (Charlotte)

Confidence and continued NBO practice may be encouraged if an NBO 'Champion' is available to provide support and continued training (Holland \& Watkins, 2015).

Some MCHNs identified time, workload and procedural restrictions as barriers to implementing the NBO. However, other MCHNs felt the restrictions to a lesser degree, perhaps due to higher confidence gained from years of NBO practice (Holland \& Watkins, 2015); “it's easy enough to do aspects of the NBO at the same time as going through a physical assessment with the baby" (Nicola). The same MCHNs also recognised the appropriateness of administering only NBO items which are aligned with infants' current states (e.g. habituation items during sleep state; Nugent et al., 2007), rather than completing all 18 items during one consultation. Consistent with Tomlin and colleagues' (2016) suggestions, MCHNs felt that their NBO practice would be less restrictive with increased managerial support and policy change.

\section{Discussion}

The present study explored MCHN's meaning-making processes when using the NBO to support infant-mother dyads. An interpretative phenomenological approach was adopted to understand MCHNs' mentalisation processes, the NBO's influence on their clinical practice, and their sense-making of experiences with dyads. MCHNs' mentalisation abilities and tendencies were evident across several themes; this began with their thought processes towards the dyads. 
They expressed that their NBO practice enabled them to view infants as unique, competent and social (Nugent, 2015). MCHNs were respectfully curious and empathic towards mothers, striving to understand the motivations behind mothers' actions. They observed the complex dyadic interactions to identify adaptive and maladaptive relational behaviour, rather than relying only on mother-centric conversations whereby the infant is often ignored or a secondary participant.

The nurses were reflective regarding their pre-NBO practice, identifying the prescriptive nature of their work, feelings of ambiguity and helplessness, and lack of focus on infants as barriers to effective support (McManus \& Nugent, 2011). MCHNs were, however, clientfocused, unanimously reporting that they chose to integrate the NBO into practice to help improve dyadic relational prospects. The MCHNs were able to navigate the complex interactions between their reflections towards the dyads with their own self-reflections; identifying areas where they could provide support through the NBO.

MCHNs expressed that their NBO practice empowered them to give voice to and advocate for the pre-verbal infants (Nugent, 2013). They were able to promote dyadic mindedness, allowing mothers a holding space to 'see' their infants as individuals with their own needs, likes, and intentions (Cologon et al., 2017; Nugent et al., 2014; Ordway et al., 2014). This appeared to promote mothers' accuracy when interpreting their infants' cues and respond more sensitively, thus promoting early infant-mother relationships. NBO practice also appeared to shift nursing support from a prescriptive approach to a collaborative one, of walking alongside the dyads as they become acquainted with one another (Ordway et al., 2014).

Overall, MCHNs stated that they derived a sense of emotional satisfaction and accomplishment when they observed dyads connect and thrive. They felt pride in their clinical practice and the opportunities that their profession provides to families. Barriers to the NBO's 
implementation were conveyed, these being isolation from other NBO practicing peers, time pressures, and prescriptive practice requirements. By way of overcoming barriers, the nurses felt that the NBO should be a formalised practice tool, to be incorporated into relevant policies and guidelines; this would provide for consistent practice and peer support (Holland \& Watkins, 2015).

\section{Limitations}

The participants who participated in this study may have been self-selecting as MCHNs who possess strong mentalisation skills, are open to professional development opportunities, and adopting a new way of working with families. Further, this study was not designed to ascertain the change in levels of mentalisation in MCHNs. However, the current findings, make it evident that MCHNs gained an added, critical dynamic to their mentalisation process: the viewpoint of the infant. MCHNs consistently reflected that pre-NBO, the focus on infants during consultations was lacking. The NBO enabled MCHNs to provide support with both infants and mothers in mind as opposed to being mother-centric. This is an important finding for a profession that aims to support infant wellbeing and development. Whilst the MCHNs may have exhibited mentalisation ability pre-NBO practice, this study indicated increased awareness and satisfaction derived from the enhanced mentalisation that the NBO appears to encourage.

\section{Implications for research and practice}

This study's findings have implications for child and family health nurses, the care they provide, their training, the families they support, and the infant mental health arena generally. MCHN support is a centrepiece of community care for parents and infants in Australia, it is, therefore, important to help practitioners avoid burnout so that their crucial service may continue. Mentalisation has been linked to feelings of accomplishment and job satisfaction, 
factors that reduce the risk of burnout (Kalliath \& Morris, 2002; Watson et al., 2016). The emotional satisfaction and professional pride reported by participants in this study supports this. MCHNs were empathic whilst maintaining a self-awareness of the separateness of their own thought processes to that of the dyads', factors which mediate emotional exhaustion (Myors et al., 2018; Slade, Sadler, Dios-Kenn, Webb, Currier-Ezepchick, \& Mayes, 2016). The NBO is a time-effective and low-cost tool that appears to decrease burnout risk and promote mentalisation in MCHNs.

Encouraging mentalisation through the NBO shifts MCHNs' practice from an authoritative model to one of collaboration, a more effective approach when building relationships with dyads and attempting to elicit behavioural change (Ordway et al., 2014). Equally important, NBO practicing MCHNs are able to provide individualised support when they become acquainted with infants' uniqueness (Nugent, 2013; Nugent et al., 2014). Previously, infants may not have been a party to the conversation, and were rendered unable to influence the caregiving they expressly need and prefer. The NBO provides a language for MCHNs to directly communicate with pre-verbal infants, a language mothers can observe and model. This process may promote positive infant-mother relationships, and gives infants opportunities to learn and demonstrate emotional regulation (Fonagy et al., 1991).

\section{Future research}

Given the explorative nature of this study, future researchers might adopt a quantitative framework to validate and extend the current findings. For example, it would be of interest to assess mentalisation before and after NBO training. There exists a current inventory of measures for both practitioner and parental mentalisation which may be employed to measure mentalisation before and after integration of NBO into practice (Fonagy et al., 1991). Exploring 
mentalisation processes in other NBO-practicing professionals (e.g. social workers, infant mental health psychologists) and other client groups (e.g. foster carers) to determine transferability of the current study's findings may also prove beneficial.

\section{Training and development}

MCHNs unanimously supported the integration of the NBO into the child and family health nursing and infant mental health training curriculum. Some MCHNs also expressed a need for forums to discuss their NBO practice. This may be in the form of peer supervision whereby practitioners are connected to one other or a group of peers. This allows practitioners to debrief, engage in peer reflective supervision, and share profession-specific practice suggestions. Nominating an NBO 'Champion' whom practitioners can consult for technical NBO advice may also be helpful (Holland \& Watkins, 2015).

\section{Conclusion}

This study suggests the NBO, a time and cost-effective tool, encourages mentalisation in practitioners and mothers. The potential benefits go beyond the quality of the consultation and the immediate dyadic benefits in the first three months. Mentalising MCHNs possess opportunities to positively steer relationships between infants and their mothers throughout infancy, thus reducing the likelihood of disrupted relationships and infant or maternal mental health concerns later in life. MCHNs in this study all advocated for universal NBO training with ongoing reflective supervision. This would follow on well from the recent incorporation of a mandatory early relational trauma-informed learning module for all MCHNs in Victoria. There is current Australian and international research testing the impact of the NBO on families and on the child and family health nursing profession and other professions working with new families. The findings of this study, if matched by positive quantitative research findings, should 
appropriately lead to policy and procedural support for NBO training and practice in community care to the benefit of both professionals and families. 


\section{References}

Barlow, J., Herath, N., Torrance, C. B., Bennett, C., \& Wei, Y. (2018). The Neonatal Behavioral Assessment Scale (NBAS) and Newborn Behavioral Observations (NBO) system for supporting caregivers and improving outcomes in caregivers and their infants. Cochrane Database of Systematic Reviews 2018, Issue 3, 1465-1858.

https://doi.org/10.1002/14651858.CD011754.pub2

Bateman, A. W., \& Fonagy, P. (2012). Handbook of Mentalizing in Mental Health Practice. Arlington, VA, US: American Psychiatric Publishing, Inc.

Beam, R. J., O’Brien, R. A., \& Neal, M. (2010). Reflective practice enhances public health nurse implementation of nurse-family partnership. Public Health Nursing, 27, 131-139. https://doi.org/10.1111/j.1525-1446.2010.00836.x

Burkhart, M. L., Borelli, J. L., Rasmussen, H. F., Brody, R., \& Sbarra, D. A. (2017). Parental mentalizing as an indirect link between attachment anxiety and parenting satisfaction, Journal of Family Psychology, 31, 203-213. https://doi.org/10.1037/fam0000270

Cologon, J., Schweitzer, R. D., King, R., \& Nolte, T. (2017). Therapist reflective functioning, therapist attachment style and therapist effectiveness. Administration and Policy in Mental Health and Mental Health Services Research, 44, 614-625. https://doi.org/10.1007/s10488017-0790-5

Davidsen, A. S. (2009). How does the general practitioner understand the patient? A qualitative study about psychological interventions in general practice. Psychology and Psychotherapy, 82, 199-217. https://doi.org/10.1348/147608308X377358

Fonagy, P., Steele, M., Steele, H., Moran, G. S., \& Higgitt, A. C. (1991). The capacity for understanding mental states: The reflective self in parent and child and its significance for 
security of attachment, Infant Mental Health Journal, 12, 201-218.

http://dx.doi.org/10.1002/1097-0355(199123)12:3<201::AID-IMHJ2280120307>3.0.CO;2-

$\underline{7}$

Garner, A. (2013). Home visiting and the biology of toxic stress: Opportunities to address early childhood adversity. Pediatrics, 132, 65-73. https://doi.org/10.1542/peds.2013-1021D

Holland, A., \& Watkins, D. (2015). Flying Start health visitors' views of implementing the Newborn Behavioural Observations: barriers and facilitating factors. Community Practitioner, 88, 33-36.

Horowitz, J. A., Murphy, C. A., Gregory, K., Wojcik, J., Pulcini, J., \& Solon, L. (2013). Nurse home visits improve maternal/infant interaction and decrease severity of postpartum depression. Journal of Obstetric, Gynecologic, \& Neonatal Nursing, 42, 287-300. https://doi.org/10.1111/1552-6909.12038

Kalliath, T., \& Morris, R. (2002). Job satisfaction among nurses: A predictor of burnout levels. The Journal of Nursing Administration, 32, 648-654. https://doi.org/ 10.1097/00005110200212000-00010

Kemp, L., Anderson, T., Travaglia, J., \& Harris, E. (2002). Sustained nurse home visiting in early childhood: Exploring Australian nursing competencies, Public Health Nursing, 22, 254259. https://doi.org/10.1111/j.0737-1209.2005.220309.x

McManus, B. M., \& Nugent, J. K. (2011). Feasibility study of early intervention provider confidence following a neurobehavioral intervention for high-risk newborns. Journal of Reproductive and Infant Psychology, 29, 395-403.

https://doi.org/10.1080/02646838.2011.623228 
Myors, K. A., Cleary, M., Johnson, M., \& Schmied, V. (2018). 'Modelling a secure-base' for women with complex needs: Attachment-based interventions used by perinatal and infant mental health clinicians, Issues in Mental Health Nursing, 39, 226-232. https://doi.org/10.1080/01612840.2017.1378784

Nicolson, S. (2015). Let's meet your baby as a person: From research to preventive perinatal practice and back again with the Newborn Behavioral Observations. Zero to Three, 36, 3539.

Nicolson, S., Carron, S., Newman, L., \& Campbell, P. (n.d.). Understanding your newborn and adapting to parenthood: A randomised controlled trial of the Newborn Behavioural Observations among first time parents at risk of maternal postnatal depression. Manuscript in preparation.

Nugent, J. K. (2013). The competent newborn and the neonatal behavioral assessment scale: T. Berry Brazelton's legacy. Journal of Child and Adolescent Psychiatric Nursing, 26, 173179. https://doi.org/10.1111/jcap.12043

Nugent, J. K. (2015). The Newborn Behavioral Observations (NBO) system as a form of intervention and support for new parents. Zero to Three, 36, 2-10.

Nugent, J. K., Bartlett, J. D., \& Valim, C. (2014). Effects of an infant-focused relationship-based hospital and home visiting intervention on reducing symptoms of postpartum maternal depression. Infants and Young Children, 27, 292-304.

https://doi.org/10.1097/iyc.0000000000000017

Nugent, J. K., Keefer, C. H., Minear, S, Johnson, L. C., \& Blanchard, Y. (2007). Understanding newborn behavior and early relationships: the Newborn Behavioral Observations (NBO) system handbook. Baltimore, MD: Brookes. 
Ordway, M. R., McMahon, T. J., Kuhn, L. D. L. H., \& Suchman, N. E. (2018). Implementation of an evidenced-based parenting program in a community mental health setting, Infant Mental Health Journal, 39(1), 92-105. http://doi.org/10.1002/imhj.21691

Ordway, M. R., Sadler, L. S., Dixon, J., \& Slade, A. (2014). Parental reflective functioning: Analysis and promotion of the concept for paediatric nursing, Journal of Clinical Nursing, 23, 3490-3500. http://doi.org/10.1111/jocn.12600

Pietkiewicz, I., \& Smith, J. (2014). A practical guide to using interpretative phenomenological analysis in qualitative research psychology. Czasopismo Psychologiczne, 18, 361-369. http://doi.org/ 10.14691/CPPJ.20.1.7

Rostad, W. L., \& Whitaker, D. J. (2016). The association between reflective functioning and parent-child relationship quality. Journal of Child and Family Studies, 25, 2164-2177. https://doi.org/10.1007/s10826-016-0388-7

Sanders, L. W., \& Buckner, E. B. (2006). The Newborn Behavioral Observations system as a nursing intervention to enhance engagement in first-time mothers: Feasibility and desirability. Pediatric Nursing, 32, 455-459.

Schilling, M., Nicolson, S., \& Ridgway, L. (2018). When mother reports panic and sadness, how might we therapeutically support mother and baby? Australian Journal of Child and Family Health Nursing, 15(1), 4-8.

Shai, D., Dollberg, D., \& Szepsenwol, O. (2017). Infant behavior and development: The importance of parental verbal and embodied mentalizing in shaping parental experiences of stress and coparenting. Infant Behavior and Development, 49, 87-96. http://doi.org/10.1016/j.infbeh.2017.08.003 
Simpson, T. E., Condon, E., Price, R. M., Finch, B. K., Sadler, L. S., \& Ordway, M. R. (2016). Demystifying infant mental health: What the primary care provider needs to know. Journal of Pediatric Health Care, 30(1), 38-48. http://doi.org/10.1016/j.pedhc.2015.09.011

Slade, A. (2006). Reflective parenting programs: Theory and development. Psychoanalytic Inquiry, 26, 640-657. http://dx.doi.org/10.1080/07351690701310698

Slade, A., Sadler, L., Dios-Kenn, C. De, Webb, D., Currier-Ezepchick, J., \& Mayes, L. (2016). Minding the Baby a reflective parenting program, The Psychoanalytic Study of the Child, 60, 74-100. https://doi.org/10.1080/00797308.2005.11800747

Smith, J. A., Flowers, P., \& Larkin, M. (2009). Interpretative phenomenological analysis: Theory, method and research. London, UK: Sage Publications.

Smith, J.A., \& Osborn, M. (2008). Interpretative phenomenological analysis. In J. A. Smith (Ed.), Qualitative Psychology: A practical guide to research methods. (2 ${ }^{\text {nd }}$ Ed, pp. 53-80). London, UK: Sage Publications.

Suchman, N. E., Ordway, M. R., Heras, L. De, \& McMahon, T. J. (2016). Mothering from the Inside Out: Results of a pilot study testing a mentalization-based therapy for mothers enrolled in mental health services. Attachment \& Human Development, 18, 596-617. http:/doi.org/10.1080/14616734.2016.1226371

Tomlin, A. M., Hines, E., \& Sturm, L. (2016). Reflection in home visiting: The what, why, and a beginning step toward how, Infant Mental Health Journal, 37, 617-627. http:/doi.org/10.1002/imhj.21610

Tomlin, A. M., Sturm, L., \& Koch, S. M. (2009). Observe, listen, wonder, and respond: A preliminary exploration of reflective function skills in early care providers. Infant Mental Health Journal, 30, 634-647. http://doi.org/10.1002/imhj.20233 
Watson, C. L., Bailey, A. E., \& Storm, K. J. (2016). Building capacity in reflective practice : A tiered model of statewide supports for local home-visiting programs, 37, 640-652. http://doi.org/10.1002/imhj.21609

Yardley, L. (2008). Demonstrating validity in qualitative psychology. In J. A. Smith (Ed.), Qualitative Psychology: A practical guide to research methods. (2 ${ }^{\text {nd }}$ ed, pp. 53-80). London, UK: Sage Publications. 